\title{
Editorial
}

\section{Studies of Bloodstream Infection Outcomes: Reading Between the Lines}

\author{
Sara E. Cosgrove, MD, MS; Yehuda Carmeli, MD, MPH
}

Understanding the origins, treatment, prevention, and outcomes of nosocomial bloodstream infections (BSIs) is of utmost importance because they are one of the most frequent and severe infectious complications of hospitalization and medical care. The outcomes of BSIs may differ depending on patient factors including underlying conditions and immune status; organism factors including virulence and resistance; and treatment factors such as agent and dose used, delay in appropriate therapy, removal of hardware, and associated supportive therapy. This issue of Infection Control and Hospital Epidemiology includes four studies that examine different factors that affect the outcome of nosocomial BSIs. ${ }^{1-4}$ These studies present an opportunity to discuss some methodologic principles in measuring the outcomes of hospital-acquired infections and how methods used in such studies may affect study results.

The design of studies that measure the effect of an infectious complication during hospitalization on the outcome of a patient is important and challenging because the many other factors that contribute to the outcome have to be neutralized. A patient who develops an infection would optimally be compared with himself or herself if no infection occurred; however, given that this is not possible, various methods of study design and analysis have been developed to adjust for factors that contribute to outcomes. One approach is to look for the "identical twin patient" in whom an infection has not occurred. This is "control by study design" in which a case-patient is matched to a controlpatient who is similar in every aspect, and the difference in outcome is compared between the patients. This approach is easy to understand and therefore has gained wide popu- larity. However, given that the twin patient never exists, the researcher must decide which factors are the most important and match on them. In our experience, if more than three factors are chosen, in most hospital-based studies it will be almost impossible to find appropriate controlpatients for many of the case-patients.

The other approach is "control by analysis" in which patients with and without infection are identified and the effect of each factor on the outcome is adjusted for by stratification or multivariable analysis. Thus, the effect of the infection on the outcome is isolated from other effects. This method requires more reliance on the skills of the statistician and the interpretation of results by the investigator and is less intuitive to the reader, who must assume that the methodology is executed correctly. With the widespread availability of user-friendly statistical packages that allow less experienced investigators to perform complex statistical analysis, this method has gained popularity, perhaps at a cost of reduction in the quality of the analyses.

Irrespective of which method is used, several issues remain important in the design of studies that examine the outcomes of patients with infections. Patients' underlying diseases and severity of illness before the infectious complication occurs are important determinates of outcome; therefore, appropriate adjustment for these factors is essential. Because severely ill patients with multiple comorbidities are more likely to develop nosocomial infections as well as to suffer adverse outcomes, failure to adjust for underlying diseases can lead to an inappropriately high estimate of the impact of an infection on patient outcomes. Investigators have employed several methods to facilitate such adjustment, including use of Acute Physiology and

Dr. Cosgrove is from the Division of Infectious Diseases, Johns Hopkins Medical Institutions, Baltimore, Maryland. Dr. Carmeli is from the Division of Infectious Diseases, Beth Israel Deaconess Medical Center, Boston, Massachusetts; and the Divisions of Infectious Diseases and Epidemiology, Tel Aviv Sourasky Medical Center, Tel Aviv, Israel.

Address reprint requests to Sara E. Cosgrove, MD, MS, The Johns Hopkins Hospital, Carnegie 284/288, 600 N. Wolfe St., Baltimore, MD 21287. 
Chronic Health Evaluation (APACHE) scores, the McCabe and Jackson severity of illness score, the chronic disease score, the active comorbidity score, the Medical Illness Severity Grouping System admission severity group score and other scoring systems designed for administrative risk adjustment, and the Charlson weighted index of comorbidity as well as enumeration of individual comorbidities. ${ }^{5}$ All methods have advantages and disadvantages; however, only the McCabe score and the APACHE score have been validated for use as predictors of infectious disease outcomes, with the latter having been validated only in the intensive care unit (ICU) setting. Moreover, comparisons between the various methods have not been studied systematically.

Lesens et al. ${ }^{1}$ take an important initial step in assessing the value of the Charlson weighted index of comorbidity as a method to control for underlying comorbidity in patients with Staphylococcus aureus bacteremia. They note that a dichotomized Charlson weighted index of comorbidity $(<3$ or $\geqslant 3)$ is a good predictor of overall mortality as well as mortality related to $S$. aureus bacteremia. The authors recognize that a major problem with the Charlson weighted index of comorbidity is that it was designed to predict mortality in oncology patients and thus does not include weights for certain diseases or disease states such as solid organ transplant, bone marrow transplant, or immune suppression that may have an impact on outcomes, especially mortality, in patients with bacteremia. Examining the use of the index as a predictor of mortality using additional data sets to provide validation of this finding would be the next step in determining the utility of the dichotomized score as a predictor of death associated with bacteremia. Nevertheless, this study provides preliminary evidence that the Charlson weighted index of comorbidity is a useful measurement of underlying comorbidity and its relationship to outcomes of patients with bacteremia.

In addition to the issue of how to measure underlying comorbidities and disease severity, another important issue is the timing of such measurement. Measurement of these variables should occur prior to development of infection because comorbidities that develop after infection has started are often attributable to infection and severity of illness may be strongly influenced by the presence of infection. These may therefore represent intermediate variables in the chain of events between the exposure (ie, the infection) and the outcome of interest (ie, mortality). ${ }^{6}$ Adjustment for an intermediate variable usually causes an underestimation of the effect of the exposure of interest on the outcome. This problem was noted previously in a study that adjusted for shock in a multivariable model that examined the risk of mortality related to methicillin-resistant $S$. aureus.? The inclusion of shock and disseminated intravascular coagulation as predictors of mortality in the multivariate analysis in the study by Lesens et al. may lead to underestimation of the predictive effect of the Charlson weighted index of comorbidity on mortality.

The issue of timing of measurement of severity of illness may also explain the results of the study by Blot et al., ${ }^{4}$ which examines the relationship between Escherichia coli BSI and mortality in patients in the ICU. This meticulously designed cohort study includes 64 ICU patients with $E$. coli BSIs who are matched by APACHE II score and principal admission diagnosis to 128 patients without $E$. coli BSIs. Control-patients were also required to have an ICU stay that was at least as long as that of the case-patient before bacteremia onset. Although one would hypothesize that bacteremia in a critically ill patient should lead to an increase in mortality, the authors find that mortality rates were similar for case-patients (43.8\%) and control-patients $(45.3 \%)$. They postulate that this finding may be related to the rapid initiation of appropriate antimicrobial therapy. This is certainly a reasonable hypothesis; however, it is somewhat implausible that rapid treatment eliminates mortality related to bacteremia. Another additional explanation for this finding is that at least $25 \%$ of the patients with bacteremia were bacteremic at the time of admission to the ICU (the lower end of the interquartile range for the average length of stay in the ICU was 1 day); thus, the authors matched on APACHE II score when at least $25 \%$ of the cases already had clinical manifestations of infection, which may bias the results to show no difference between affected and nonaffected patients.

Another approach to controlling for underlying illness and the effects that it has on outcomes is to restrict the analysis to a relatively homogeneous population. Both Wisplinghoff et al. ${ }^{3}$ and Kim et al. ${ }^{2}$ assess outcomes of BSI in neutropenic patients and provide important information about its implications. Wisplinghoff et al. studied the outcomes of 81 neutropenic patients with hematologic malignancies who developed BSIs with a variety of pathogens, including coagulase-negative staphylococci $(22 \%), E$. coli $(19 \%)$, viridans group streptococci $(17 \%)$, other streptococci $(17 \%)$, Pseudomonas aeruginosa (7\%), and $S$. aureus $(6 \%)$, and found a rate of $14.3 \mathrm{BSIs}$ per 100 neutropenic episodes. These patients were matched to control-patients who did not develop BSIs on gender, underlying malignancy, duration and severity of neutropenia, and radiation therapy. Portions of the group were also matched on length of stay prior to infection, age ( \pm 5 years), and stage of illness. Mortality was higher for P. aeruginosa $(50 \%)$ and $S$. aureus $(40 \%)$ than for other pathogens, and the attributable mortality rate was $12 \%$. The excess length of stay attributable to BSI was 9 days and the excess cost was $\$ 3,170$ per patient.

Wisplinghoff et al. adjust for underlying patient differences using matching (control by study design). Although this approach may provide reasonable estimates of outcomes in this study because of the similarities of the patients to start (all neutropenic patients with hematologic malignancies), it does limit the authors' ability to control for other potential confounding factors such as source of BSI, ICU admission, and underlying comorbidities that might influence outcomes. A hybrid approach of performing a matched study with a matched multivariate analysis may improve the ability to control for confounding and would add further strength to the study findings. 
Outcomes associated with infection should be counted only after the infection has started. Thus, when assessing the length of stay and cost associated with BSI, the length of stay and cost accrued before BSI should be subtracted from the total. Matching on length of stay prior to infection can control for preinfection hospital-days, but may not control well for costs accrued before infection. As Wisplinghoff et al. were not able to match on length of stay prior to BSI for every patient, the actual attributable length of stay and cost estimates in their cohort may be somewhat lower than those reported.

Although not related to a methodologic principle, the interpretation of the results of the study by Kim et al. merits comment. Kim et al. performed a retrospective analysis of the outcomes of neutropenic cancer patients with $S$. aureus catheter-related BSI in whom salvage of the catheter without removal was attempted. The authors studied 32 episodes of $S$. aureus bacteremia in which there were 24 catheter salvage attempts and found that the overall success rate was $38 \%$. Among patients who underwent salvage attempts, those with negative follow-up blood cultures 2 to 3 days after therapy were more likely to have a successful salvage attempt than were those with positive follow-up blood cultures ( $65 \%$ vs $14 \%$ ). Such information is important for the healthcare provider because it provides prognostic information that can be used to aid in decision making and in discussions with patients. Although this study may lead providers to consider salvage attempts in patients without evidence of sepsis who have negative follow-up blood cultures 2 to 3 days after the initiation of antistaphylococcal therapy, it is important to acknowledge that $18 \%$ of the patients in this group died as a consequence of $S$. aureus infection and $12 \%$ had recurrent bacteremia and its associated morbidity. This high morbidity and mortality even in the "lowest risk" group should prompt careful consideration of the ramifications of catheter salvage attempts in the neutropenic population.

The measurement of outcomes associated with nosocomial BSI is important to both providers, who can use the data to understand the prognosis of the patients whom they are treating, and institutions, which can use the informa-. tion to allocate resources directed at the prevention of BSI appropriately. Thus, additional studies are needed to both assess outcomes of BSI and develop and better define appropriate methodology.

\section{REFERENCES}

1. Lesens $\mathrm{O}$, Methlin $\mathrm{C}$, Hansmann $\mathrm{Y}$, et al. Role of comorbidity in mortality related to Staphylococcus aureus bacteremia: a prospective study using the Charlson weighted index of comorbidity. Infect Control Hosp Epidemiol 2003;24:890-896.

2. Kim S, Kang C, Kim H, et al. Outcomes of Hickman catheter salvage in febrile neutropenic cancer patients with Staphylococcus aureus bacteremia. Infect Control Hosp Epidemiol 2003;24:897-904.

3. Wisplinghoff $\mathrm{H}$, Cornely OA, Moser $\mathrm{S}$, et al. Outcomes of nosocomial bloodstream infections in adult neutropenic patients: a prospective cohort and matched case-control study. Infect Control Hosp Epidemiol 2003:24:905-911.

4. Blot S, Vandewoude $\mathrm{K}$, Hoste $\mathrm{E}$, et al. Absence of excess mortality in critically ill patients with nosocomial Escherichia coli bacteremia. Infect Control Hosp Epidemiol 2003;24:912-915.

5. Cosgrove SE, Carmeli Y. The impact of antimicrobial resistance on health and economic outcomes. Clin Infect Dis 2003;36:1433-1437.

6. Rothman KJ, Greenland S. Modern Epidemiology. Philadelphia: Lippincott Williams \& Wilkins; 1998.

7. Perencevich EN. Excess shock and mortality in Staphylococcus aureus related to methicillin resistance. Clin Infect Dis 2000;31:1311-1313. 